\section{Educación sexual en las carreras de la salud del Campus Eloísa Díaz de la Universidad de Chile}

\author{
RAMIRO MOLINA C. ${ }^{1}$, SEBASTIÁN ALARCÓN CH.,a, \\ TEMÍSTOCLES MOLINA G. ${ }^{3, \mathrm{~b}}$
}

\section{Evaluation of a general education course on sexuality for university students}

Background: Education on human sexuality was instituted in Eloisa Díaz Campus in 1946. From 2018 an online self-learning general education course on sexuality was started. Aim: To know the degree of knowledge about sexuality and the perception about the course of 586 students ( $42 \%$ women) who approved the course. Material and Methods: The Myths and False beliefs and Self-perception on sexuality tests were applied to students at the onset and the end of the course. They also anonymously evaluated the course. Results: In the first assessment, the proportion of correct answers about sexuality and reproduction were 47 and $40 \%$ respectively. In the second assessment, 70\% of respondents had open criteria about sexuality and $90 \%$ rejected traditional criteria. In the anonymous assessment, 95\% estimated that the objectives of the course were accomplished. Its quality was considered good or excellent by 95\% of respondents. Conclusions: This course was well accepted by students and corrects their knowledge gaps in sexuality. Despite the social unrest during 2019 and the pandemic during 2020, the course was successfully completed by most students.

(Rev Med Chile 2021; 149: 447-457)

Key words: Faculty, Medical; Learning; Sex Education.
'Departamento de Salud Pública, Departamento de Obstetricia y Ginecología Norte. Facultad de Medicina. Universidad de Chile. Santiago, Chile.

${ }^{2}$ Departamento de Salud Pública, Unidad de Tics. Facultad de Medicina Universidad de Chile.

Santiago, Chile.

${ }^{3}$ Centro de Medicina

Reproductiva y desarrollo Integral de la Adolescencia (CEMERA).

Ingeniero en Computación e informática. Profesor de Estado educación Técnico Profesional.

bEstadístico, Magister en Estadística.

Recibido el 23 de marzo de 2020, aceptado el 26 de enero de 2021.

Correspondencia a: Ramiro Molina Cartes Managua 2222 Dep 151. Ñuñoa. Santiago. Chile. rammolinar@gmail.com
U NESCO, describe la Educación Sexual Integral (ESI) como la preparación de las personas para su seguridad, productividad y vida plena, basadas en evidencias científicas publicadas, conocimientos precisos y apropiados, actitudes, habilidades, valores positivos, respeto por los derechos humanos, igualdad y diversidad de género, actitudes y destrezas que contribuyen a tener relaciones interpersonales más seguras, saludables y positivas. Además ayuda a jóvenes ( 10 a 24 años), a reflexionar sobre las normas sociales, los valores culturales y las creencias tradicionales para comprender y manejar en forma más adecuada sus relaciones con otros pares, padres, profesores $\mathrm{y}$ otros adultos de su comunidad ${ }^{1}$.

La Conferencia Internacional de Población y
Desarrollo define la Salud Reproductiva $(1994)^{2}$. En 2013, OMS, publica con varias agencias de NN.UU, lo definido como Salud Sexual y Reproductiva en Adolescentes (SRHA) ${ }^{3}$.

Europa define el rol de la Educación en sexualidad, en el contexto de la Salud Sexual y Reproductiva Adolescente y recomienda su inclusión y desarrollo en el sistema escolar de cada país ${ }^{4}$. En 2009 y 2016 UNESCO publica las evidencias de la Educación Sexual ${ }^{5,6}$.

Chile no tiene un programa de educación sexual escolar. En 2010, publica la ley 20.418: Información, Orientación y Prestaciones en Materia de Regulación de la Fertilidad. Un párrafo del $1^{\text {er }}$ artículo hace obligatoria la enseñanza de educación sexual a partir de la enseñanza media. 
Ministerio de Salud, publica su Reglamento, (2013), sin incluir la Educación Sexual ${ }^{7,8}$.

Entre 2011 y 2014 el Ministerio de Educación y SERNAM implementan un programa de Educación Sexual y Afectividad aplicado por 7 instituciones: Universitarias y ONG's. Su aplicación fue evaluada en $2019^{9}$.

La Comisión de Educación de la Cámara de Diputados en su $20^{\circ}$ Sesión, 2019, modifica la ley 20.418 para implementar programas de educación sexual, afectividad y género desde el segundo nivel de transición de la educación parvularia ${ }^{10}$. No es aprobado por la Cámara de Diputados, el 15 de octubre $2020^{11}$.

La Universidad de Chile fundada en 1842, es la más antigua e importante universidad Pública del país y su Facultad de Medicina tiene la mayor cantidad de estudiantes de pre y de postgrado. Sus estudiantes provienen de la educación pública, generalmente $^{12}$.

En las carreras de la Salud, la educación sexual es importante para su ejercicio profesional comunitario. Es materia de interés en la mayoría de los países desarrollados como en desarrollo ${ }^{13-15}$. Los conceptos de ESI se profundizan, en áreas específicas de las Carreras de Salud ${ }^{16-18}$. Las opiniones de estudiantes de medicina en estas materias son exploradas a nivel internacional ${ }^{19-23}$.

La enseñanza de la Sexualidad Humana en la Facultad de Medicina de la Universidad de Chile ha tenido un lento desarrollo. El Prof. Dr. Waldemar Coutts en la cátedra de urología desarrolló cursos de sexología hasta $1946^{24}$. En los 60', en el Curso de Ginecología de VI año, el Prof. Osvaldo Quijada Cerda, dictaba una clase acerca de Sexualidad Humana ${ }^{25}$. En 1969, a solicitud de estudiantes de Medicina de VI año, se desarrolla un curso de Sexualidad Humana con Docentes de: Salud Pública, Obstetricia /Ginecología, Urología, Psiquiatría, Medicina Legal y Pediatría. Desde 1971, la Dra. Inés Krausz del Departamento de Salud Pública, dictó, 2 veces al año, un curso de Sexología para estudiantes de VI año de Medicina. En 1981, el Departamento de Obstetricia y Ginecología Norte inicia actividades clínicas y de investigación en embarazo adolescente y nace el Centro de Medicina y Desarrollo Integral de la Adolescencia, (CEMERA), donde se detectó la ausencia total de educación sexual en adolescentes embarazadas. Se inicia un plan de Educación Sexual Integral Escolar capacitando Profesores de
Básica y Media, según recomienda UNESCO ${ }^{26-29}$. En 1995-1996, CEMERA dicta un curso electivo: Salud Sexual y Reproductiva para Carreras de la Salud. Desde 2001 a 2012, se explora diferentes técnicas de capacitación docente, con cursos presenciales, parcialmente presenciales y no presenciales: vía interactiva por internet ${ }^{30-32}$. En 2015 y 2016, se instala en la Escuela de Salud Pública, Unidad de TIC's para Innovación Educativa (UTIE), un sitio WEB abierto y gratuito: Curso de Educación Sexual On Line de Auto Aprendizaje (CESOLAA). Se inaugura en enero de 2017: www.educacionsexual.uchile.cl, compartiendo la docencia con el Departamento de Obstetricia y Ginecología Norte ${ }^{33,34}$. En diciembre de 2017, por solicitud de la Vice-Decanatura de la Facultad de Medicina, se instala como Curso semestral de Formación General (CFG) para las 8 Carreras de la Salud de la Facultad. Se desarrolla en 2018/2019.

¿Qué ha demostrado la evaluación de esta experiencia?, ¿Es replicable?

\section{Material y Método}

\section{Material del curso de educación sexual}

1. Libro virtual: 17 capítulos y Audiolibro virtual: habilidades visuales diferentes.

2. 14 Talleres-Videos didácticos con lenguaje de señas.

3. 17 clases de 11 docentes con 17 Sets de Diapositivas.

4. 3 Películas profesionales originales: adolescente embarazada.

5. 1 película chilena: La Mujer Fantástica, como apoyo didáctico a capítulo XVII.

6. 17 Sets de preguntas de evaluación y 14 Sets de preguntas de talleres-videos.

\section{Método}

\section{Aspectos generales}

La Facultad de Medicina informa semestralmente de los 20 a 30 CFG disponibles. Es obligatorio elegir $1 \mathrm{CFG}$ semestral de 52 horas directas/27 indirectas. Aporta 2 créditos.

\section{Aspectos específicos}

\section{Inscripción}

En el sitio: www.educacionsexual.uchile.cl, 
Curso Cerrado: Educación Sexual online Medicina, (CESOMED).

\section{Desarrollo}

12 a 14 semanas (Esquema 1).

\section{Contenidos}

1) Educación Sexual con referencia a Chile.

2) Metodología en educación Sexual.

3) Desarrollo Psicosexual Normal.

4) Pubertad Normal.

5) Adolescencia y Juventud.

6) Diversidad Sexual, en la Adolescencia y Juventud.

7) Afectividad y Sexualidad.

8) Característica de la sexualidad y respuesta sexual humana.

9) Adolescente embarazada.

10)Aborto, Mitos y Realidades.

11)Infecciones de Transmisión Sexual.

12) VIH y SIDA.

13)Anticoncepción.

14)Conceptos de Sexualidad y Roles de Género.

15)Acoso y Abuso Sexual.

16)Familia y Tipos de Organización.

17)Transexualidad.

14 Videos-Talleres: Elaborados y filmados en el Centro de Habilidades Clínicas y editados en Unidad Audiovisual del Departamento de Educación de la Facultad de Medicina.

\section{Participantes}

Estudiantes de pregrado de 8 carreras, Facultad de Medicina. Hubo 15 estudiantes de otras Facultades, Campus Eloísa Díaz.

\section{Evaluación del Curso}

Evaluación previa: dos test: (a) 13 afirmaciones de verdadero/falso, basadas en mitos y falsas creencias (b) 9 criterios de auto percepción y de conductas en sexualidad humana ${ }^{35}$. Ambos test probados y evaluados en: Un estudio de cohorte da casos y controles en escolares de $7^{\text {to }}$ a $4^{\text {to }}$ me$\operatorname{dio}^{36}$ y desde 1998 a 2011 en encuestas de cursos a profesores en $\mathrm{Chile}^{37}$.

La evaluación final mide: A) Percepción del cumplimiento de los objetivos de 5 Módulos y B) Calificación cualitativa basada en 4 criterios recomendados por la Facultad de Medicina: Relevancia de Contenidos, Nivel Respeto y Claridad en la exposición de temas, Grado de organización de la información y Resumen de la Calificación de contenidos. El análisis estadístico usó Intervalos de Confianza ${ }^{38}$.

\section{Resultados}

\section{Características de la Población Estudiantil}

En cuatro semestres: 621 estudiantes de Carreras de la Salud: Medicina: 32,37\%, Tecnología Médica:16,75\%, Obstetricia: 12,24\%, Terapia Ocupacional 10,63\% y Enfermería 8,05\%. Aprobó 94,36\% y No aprobó 5,64\%. Además, 12 estudiantes de Facultad de Odontología y 3 de Facultad de Química y Farmacia. Total 636 inscritos de los cuales aprobaron $601(94,5 \%)$.

Fueron $41,5 \%$ femenino, 58,2\% masculino y 0,3\% Otro. Información, no incluida en Tabla 1 . Sin diferencias significativas por género.

\begin{tabular}{|l|c|c|c|c|c|}
\hline $\mathbf{5}$ MóduLOS & M1 & M2 & M 3 & M 4 & M 5 \\
\hline 17 CAPÍTULOS & 3 & 3 & 2 & 4 & 5 \\
\hline 17 CLASES & 2 & 4 & 2 & 3 & 5 \\
\hline 14 VIDEO-TALLERES & 2 & 3 & 2 & 3 & 4 \\
\hline 4 PELÍCULAS & & & 3 & & 1 \\
\hline 5 FOROS VIRTUALES & 1 & 1 & 1 & 1 & 1 \\
\hline 17 EVALUACIONES CAPÍTULOS & 3 & 3 & 2 & 4 & 5 \\
\hline 14 EVALUACIONES VIDEOS-TALLERES & 2 & 3 & 5 & 2 & 2 \\
\hline 65 DÍAS HÁBILES & 9 & 13 & 11 & 15 & 17 \\
\hline
\end{tabular}

Esquema 1. Distribución de los 5 módulos de CESOMED. 
Tabla 1. Estudiantes de Carreras de la Salud de la Facultad de Medicina y otras Carreras de Campus Eloísa Díaz. Universidad de Chile, inscritos en el Curso de Formación General: Educación Sexual On Line de Auto Aprendizaje, en los 4 semestres de 2018 y 2019. Resultados finales

\begin{tabular}{|c|c|c|c|c|c|}
\hline $\begin{array}{l}\text { Estudiantes de Pregrado/ Postg. } \\
\text { Carreras Facultad de Medicina }\end{array}$ & 2018 & 2019 & Total & $\%$ & $\begin{array}{l}\chi^{2} \\
p\end{array}$ \\
\hline Enfermería & 24 & 26 & 50 & 8,05 & \multirow{9}{*}{$0,892^{* *}$} \\
\hline Fonoaudiología & 13 & 21 & 34 & 5,47 & \\
\hline Kinesiología & 17 & 20 & 37 & 5,96 & \\
\hline Medicina & 89 & 112 & 201 & 32,37 & \\
\hline Nutrición & 8 & 10 & 18 & 2,89 & \\
\hline Obstetricia & 27 & 49 & 76 & 12,24 & \\
\hline Tecnología médica & 44 & 60 & 104 & 16,75 & \\
\hline Terapia ocupacional & 27 & 39 & 66 & 10,63 & \\
\hline Total aprobados Medicina* & 249 & 337 & 586 & 94,36 & \\
\hline No aprobados F. Medicina*** & 19 & 16 & 35 & 5,64 & \\
\hline Total Facultad Medicina & 268 & 353 & 621 & 100 & \\
\hline Facultad Odontología aprobados & 6 & 6 & 12 & 80 & \\
\hline Facultad Química y Farmacia aprobados & 2 & 1 & 3 & 20 & \\
\hline Total otras Facultades* & 8 & 7 & 15 & 100 & \\
\hline Total de estudiantes aprobados* & 257 & 360 & 601 & & \\
\hline Gran total de estudiantes & 276 & 360 & 636 & & \\
\hline
\end{tabular}

*Total de Estudiantes que evalúan el Curso (601). **Por Carrera No hubo diferencias estadísticamente significativas en el № de aprobados, según el número de inscritos por año en la Facultad de Medicina. ***Estudiantes No Aprobados. No incluidos en la evaluación.

Hubo $19(7,1 \%)$ y $16(4,5 \%)$, estudiantes de la Facultad de Medicina que no aprobaron en 2018 y 2019. Sin diferencias significativas por carrera.

\section{Evaluación inicial: Conocimientos Básicos y} Mitos/Falsas creencias en Sexualidad Humana

De las 13 afirmaciones: 5 son de Reproducción Humana, todas incorrectas: $(2,4,5,8$ y 11) y 8 de Sexualidad (1, 3, 6, 7, 9, 10, 12 y 13), de las cuales 3 son correctas: $(1,9$ y 10$)$ y 5 : incorrectas $(3,6,7$, 12 y 13). Las respuestas de los estudiantes totales fueron: $44,1 \%$ correctas y $55,9 \%$ incorrectas, cuyo intervalo de confianza de $95 \%$, fue de 40,1 a $48,1 \%$ de respuestas correctas (Tabla 2).

El análisis de esta Tabla 2, se obtiene del Esquema 2, en el cual las 5 preguntas de Reproducción Humana (2,4,5,8 y 11), alcanzan 39,5\% de respuestas correctas y $60,5 \%$ de respuestas incorrectas. Las 8 preguntas de Sexualidad: $(1,3$, $6,7,9,10,12$ y 13 ) alcanzaron $47 \%$ de respuestas correctas y $53 \%$ de incorrectas.
La experiencia en 22 cursos aplicados en profesores de enseñanza Básica y Media (1992-1996) ${ }^{37}$, obtuvo 99,5\% de correctas en los test finales de los cursos, tanto de Sexualidad como de Reproducción y en el estudio de casos y controles en Escolares de $7^{0}$ Básico a $4^{\text {to }}$ Medio (1994-1995) ${ }^{36}$, se llegó a 72,2\% de correctas en los test finales de los casos intervenidos con educación. Dadas estas evidencias, ya no se aplica estos test validados, al final de los actuales cursos.

Un segundo enfoque exploratorio de autopercepción y conductas en sexualidad humana, en la Tabla 3. Las primeras 6 afirmaciones, muestran una población con criterios amplios. Los criterios más abiertos superan el $70 \%$ de población encuestada.

Las afirmaciones de religiosidad: 7, 8 y 9; alcanzan las mayores proporciones de: "Sin Opinión", sobre un quinto de la población que responde. La pregunta 7 , tradicionalmente muy específica, revela $94,1 \%$ de Desacuerdo y Sin opinión. Pre- 
Tabla 2. Resultados del test inicial de conocimiento: Mitos y Falsas creencias en sexualidad humana (93,9\% de respuestas de estudiantes al inicio del curso*)

\begin{tabular}{|c|c|c|c|c|c|c|c|}
\hline \multirow{3}{*}{$\begin{array}{l}\text { Áreas de afirmaciones del test } \\
\text { Sexualidad (S), Reproducc. Humana (RH) } \\
\text { Correctas (C) e Incorrectas (I) }\end{array}$} & \multicolumn{6}{|c|}{ Respuesta de estudiantes } & \multirow{3}{*}{$\begin{array}{c}\% \text { de } \\
\text { respuestas } \\
\text { Correctas } \\
\text { Intervalo } \\
\text { Confianza } \\
(I C)^{* *}(95 \%)\end{array}$} \\
\hline & \multicolumn{2}{|c|}{ Correctas } & \multicolumn{2}{|c|}{ Incorrectas } & \multicolumn{2}{|c|}{$\begin{array}{l}\text { Total } \\
\text { respuestas* }\end{array}$} & \\
\hline & $\mathbf{n}$ & $\%$ & $\mathbf{n}$ & $\%$ & $\mathbf{n}$ & $\%$ & \\
\hline $\begin{array}{l}\text { 1. La masturbación frecuente en adolescentes puede } \\
\text { provocar sentimientos de culpabilidad, pero no necesa- } \\
\text { riamente daños fisiológicos (S), (C) }\end{array}$ & 319 & 53,4 & 278 & 46,6 & 597 & 100 & $(49,3-57,5)$ \\
\hline $\begin{array}{l}\text { 2. Un hombre dotado de pene pequeño tendrá dificulta- } \\
\text { des para engendrar y para satisfacer a su pareja }(\mathrm{RH}),(\mathrm{I})\end{array}$ & 222 & 37,2 & 375 & 62,8 & 597 & 100 & $(33,3-41,2)$ \\
\hline $\begin{array}{l}\text { 3. Los niños carecen de manifestaciones de sexualidad } \\
\text { pues son propias de la adolescencia y la adultez (S), (I) }\end{array}$ & 251 & 42,0 & 346 & 58,0 & 597 & 100 & $(38,0-46,1)$ \\
\hline $\begin{array}{l}\text { 4. La mujer anorgásmica difícilmente tendrá posibilidades } \\
\text { de embarazarse }(\mathrm{RH}),(\mathrm{I})\end{array}$ & 214 & 35,8 & 383 & 64,2 & 597 & 100 & $(32,0-39,8)$ \\
\hline $\begin{array}{l}\text { 5. La joven adolescente que inicia actividad sexual sin pro- } \\
\text { tección, sólo en la primera relación, tiene la seguridad } \\
\text { de no quedar embarazada }(\mathrm{RH}),(\mathrm{I})\end{array}$ & 206 & 34,5 & 391 & 65,5 & 597 & 100 & $(30,7-38,5)$ \\
\hline $\begin{array}{l}\text { 6. Un par de experiencias homosexuales en la adolescencia } \\
\text { pueden determinar homosexualidad en el futuro (S), (I) }\end{array}$ & 231 & 38,7 & 366 & 61,3 & 597 & 100 & $(34,8-42,7)$ \\
\hline $\begin{array}{l}\text { 7. En la Tercera edad se pierde la capacidad física y psíqui- } \\
\text { ca para una adecuada y plena sexualidad (S), (I) }\end{array}$ & 256 & 42,9 & 341 & 57,1 & 597 & 100 & $(38,9-47,0)$ \\
\hline $\begin{array}{l}\text { 8. El desgarramiento del himen significa que se ha iniciado } \\
\text { la actividad sexual }(\mathrm{RH}),(\mathrm{I})\end{array}$ & 228 & 38,2 & 369 & 61,8 & 597 & 100 & $(34,3-42,2)$ \\
\hline $\begin{array}{l}\text { 9. No todos los que adquieren gonorrea, manifiestan los } \\
\text { síntomas (S), (C) }\end{array}$ & 337 & 56,4 & 260 & 43,6 & 597 & 100 & $(52,4-60,5)$ \\
\hline $\begin{array}{l}\text { 10. El aborto aunque se legalice, no representa un verdade- } \\
\text { ro método de planificación familiar (S), (C) }\end{array}$ & 314 & 52,6 & 283 & 47,4 & 597 & 100 & $(48,5-56,7)$ \\
\hline $\begin{array}{l}\text { 11. Para que se produzca un embarazo es requisito indis- } \\
\text { pensable que haya penetración }(\mathrm{RH}),(\mathrm{I})\end{array}$ & 308 & 51,6 & 289 & 48,4 & 597 & 100 & $(47,5-55,7)$ \\
\hline $\begin{array}{l}\text { 12. Alcanzar el orgasmo simultaneo, es el objetivo central } \\
\text { de una pareja madura y estable (S), (I) }\end{array}$ & 253 & 42,4 & 344 & 57,6 & 597 & 100 & $(38,4-46,5)$ \\
\hline $\begin{array}{l}\text { 13. La abstinencia periódica, es una opción que necesa- } \\
\text { riamente perjudicará la relación de pareja, tanto física } \\
\text { como afectivamente }(S),(I)\end{array}$ & 284 & 47,6 & 313 & 52,4 & 597 & 100 & $(43,5-51,7)$ \\
\hline $\begin{array}{l}\text { Total } 8 \text { de Sexualidad (S); } 5 \text { de Reproducción Humana (RH) } \\
\text { Total } 5 \text { respuestas Correctas }(\mathrm{C}) ; 8 \text { respuestas Incorrectas (I) }\end{array}$ & 3.423 & 44,1 & 4.338 & 55,9 & 7.761 & 100 & $(40,1-48,1)$ \\
\hline
\end{tabular}

*De los 636 estudiantes iniciales, 597 contestaron la encuesta inicial. **IC: Rango probable de estudiantes que responderán al concepto esperado de Correcto o Incorrecto.

\begin{tabular}{|l|c|c|c|}
\hline & CORRECTA & INCORRECTA & TOTAL \\
\hline SEXUALIDAD & 2.245 & 2.531 & 4.776 \\
8 PREGUNTAS & $47 \%$ & $53 \%$ & $100 \%$ \\
\hline REPRODUCCIÓN & 1.178 & 1.807 & 2.985 \\
5 PREGUNTAS & $39,5 \%$ & $60,5 \%$ & $100 \%$ \\
\hline \multicolumn{4}{|r}{} \\
\hline
\end{tabular}

Esquema 2. Test Inicial en Sexualidad y Reproducción. 


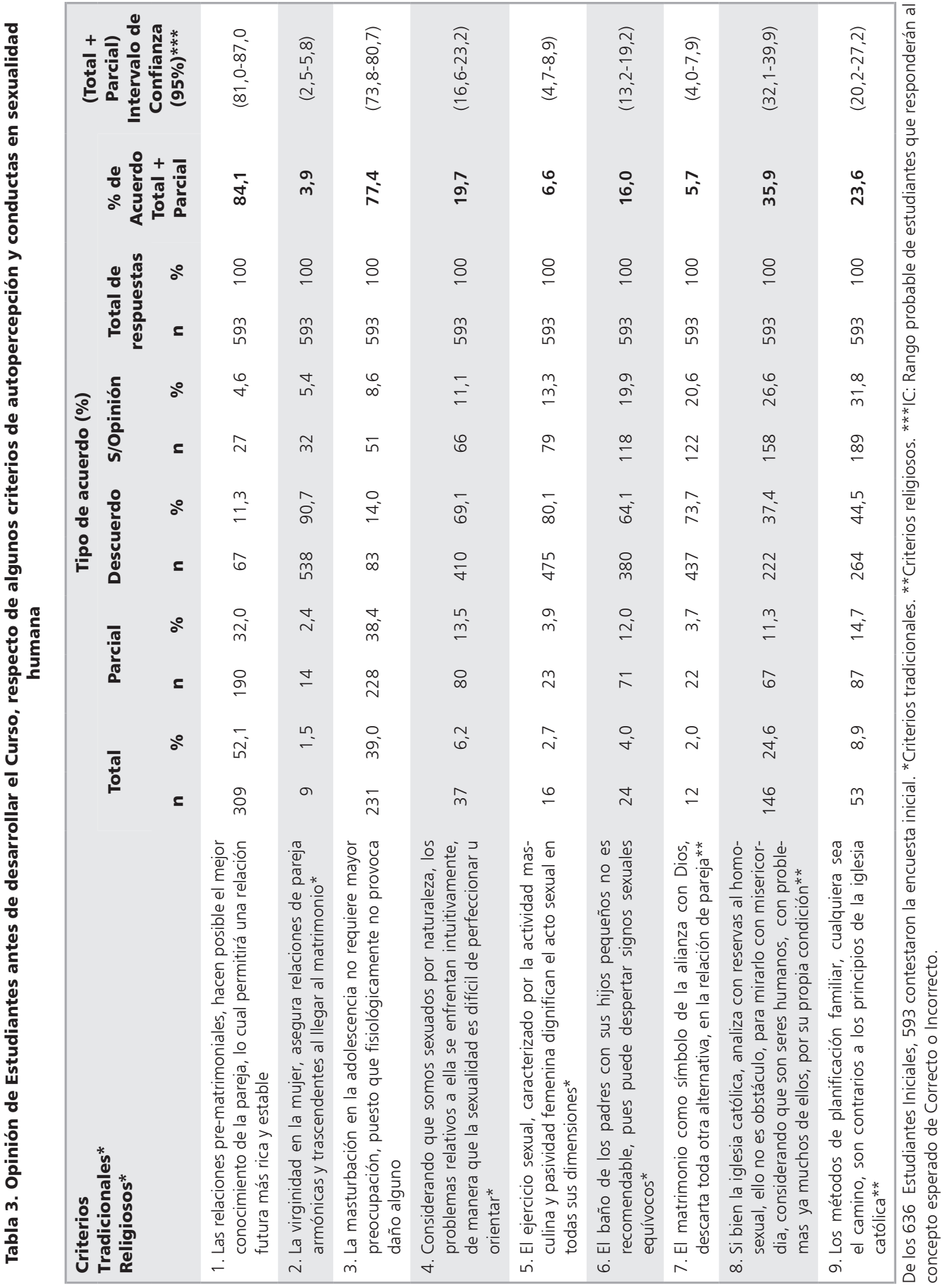


gunta 8 alcanza 37,4\% de Desacuerdo y 35,9\% en Acuerdo Total o Parcial, y una proporción alta de: Sin Opinión, posiblemente es un tema menos explorado, pero se encuentra dentro del límite de confianza. La Pregunta 9, muy tradicional, es respondida en $76 \%$ de Acuerdo o Sin Opinión y casi 1/4 de la población como Acuerdo Total o Parcial, tal vez por falta de información o desinterés en una determinada ideología. Los criterios Tradicionales y Religiosos tienen baja o muy baja proporción de Intervalos de Confianza.

\section{Evaluación de los estudiantes acerca de la Calidad del Curso}

El Cumplimiento de los Objetivos de 5 módulos con una escala de calidad, en 5 tramos, reveló un cumplimiento Bueno y Excelente de los objetivos: entre 93\% a 96\%. Las respuestas Bueno y Excelente es 94,9\%. Significativa en los 5 Módulos. El Estallido Social en el País, durante el segundo semestre de 2019 que afectó la docencia, no se reflejó en el promedio del IC, fue 93,2 a 96,5\% (Tabla 4).

En 2019 hubo un capítulo más y explica el mayor número de respuestas en comparación a 2018. Los Criterios de Calidad: ${ }^{\star}$ Relevancia de los Contenidos, ${ }^{\star}$ Exposición Respetuosa y Clara, ${ }^{*}$ Calidad de la Organización para el aprendizaje y
* Resumen de Calidad de los Contenidos, alcanzan un promedio de 95,35\% como Bueno y Excelente. (Tabla 5). En 2019 aumentó la proporción de Excelente en los 4 criterios que coincide con la actualización de 5 capítulos y clases por diferentes docentes, en 9 capítulos. El I.C. promedio de los cuatro criterios fue $95,4 \%$, con una variación entre 91,4\% en los criterios de Exposición Respetuosa y Clara en 2018, a 98,1\% en Resumen de Calificación de Contenidos en 2019. El promedio final de los criterios Deficiente e Insuficiente fue 1,22\%.

\section{Discusión}

Esta experiencia cubre las falencias de educación sexual escolar en Chile y se justifica al observar la exploración inicial en conocimientos, mitos y falsas creencias, que hubo menos de 50\% de respuestas correctas esperadas con IC de 40,1 a $48,1 \%$.

En el test inicial de conocimientos: Autopercepción y Conductas en sexualidad en los 4 cursos, reveló que los estudiantes tienen criterios, culturalmente menos tradicionales, aunque no alcanzan el máximo, como sería esperable en futuros profesionales del área de la Salud. La religiosidad tuvo baja influencia. Pero, predomina la percep-

Tabla 4. Evaluación de los estudiantes del Grado de Cumplimiento de objetivos del curso por módulos en 4 semestres 2018 y 2019

\begin{tabular}{|c|c|c|c|c|c|c|c|c|c|c|c|c|c|c|}
\hline \multirow[t]{2}{*}{ Módulos } & \multicolumn{2}{|c|}{ Deficiente } & \multicolumn{2}{|c|}{ Insuficiente } & \multicolumn{2}{|c|}{ Regular } & \multicolumn{2}{|c|}{ Bueno } & \multicolumn{2}{|c|}{ Excelente } & \multicolumn{2}{|c|}{ Total } & \multirow{2}{*}{$\begin{array}{c}\% \\
\text { Bueno + } \\
\text { Excelente }\end{array}$} & \multirow{2}{*}{$\begin{array}{c}\text { Bue + Excel } \\
\text { Intervalo de } \\
\text { Confianza } \\
(95 \%)^{* *}\end{array}$} \\
\hline & $n$ & $\%$ & $n$ & $\%$ & $\mathbf{n}$ & $\%$ & $\mathbf{n}$ & $\%$ & n & $\%$ & $\mathbf{n}$ & $\%$ & & \\
\hline $1^{\text {ero }}$ & 7 & 1,2 & 9 & 1,6 & 23 & 4,0 & 144 & 25 & 394 & 68,2 & 577 & 100 & 93,2 & $(90,9-95,1)$ \\
\hline $2^{\text {do }}$ & 1 & 0,2 & 1 & 0,2 & 16 & 2,9 & 108 & 19,3 & 435 & 77,4 & $561 *$ & 100 & 96,8 & $(98,0-98,1)$ \\
\hline $3^{\text {ero }}$ & 3 & 0,6 & 5 & 1,0 & 18 & 3,5 & 111 & 21,7 & 374 & 73,2 & $511 *$ & 100 & 94,9 & $(92,6-96,6)$ \\
\hline $4^{\text {to }}$ & 2 & 0,4 & 2 & 0,4 & 16 & 3,4 & 95 & 20,2 & 356 & 75,6 & $471 *$ & 100 & 95,8 & $(93,5-97,4)$ \\
\hline $5^{\text {to }}$ & 0 & 0 & 3 & 0,6 & 31 & 6,0 & 140 & 27,1 & 342 & 66,3 & $516^{*}$ & 100 & 93,4 & $(90,9-95,4)$ \\
\hline Total & 13 & 0,4 & 20 & 0,8 & 104 & 3,9 & 598 & 22,7 & 1.901 & 72,2 & 2.636 & 100 & 94,9 & $(93,2-96,5)$ \\
\hline \multirow[t]{2}{*}{ Extremos } & \multicolumn{4}{|c|}{33} & \multicolumn{2}{|c|}{104} & \multicolumn{4}{|c|}{2.499} & \multicolumn{2}{|c|}{2.636} & & \\
\hline & \multicolumn{4}{|c|}{$1,2 \%$} & \multicolumn{2}{|c|}{$3,9 \%$} & \multicolumn{4}{|c|}{$94,9 \%$} & \multicolumn{2}{|c|}{100} & & \\
\hline
\end{tabular}

*De los 601 estudiantes aprobados, 577 contestaron la encuesta final. Los módulos $2^{\text {do }}, 3^{\text {ero }}, 4^{\text {to }}$ y $5^{\text {to }}$ fueron afectados durante el segundo semestre de 2019 por el estallido social del 18 de octubre. El $2^{\text {do }}$ semestre 2019 se posterga hasta fines de enero de 2020. La encuestas finales son anónimas /voluntarias, luego de terminado cada módulo. ${ }^{* *} \mid \mathrm{C}$ : Rango probable de estudiantes que responden al concepto de Bueno+ Excelente. 
Tabla 5. Evaluación de Calidad del Desarrollo del Curso por las y los estudiantes. Incluyen los $\mathbf{5}$ módulos y 16 capítulos en 2018 y 17 capítulos en 2019. Registro de las respuestas en cada criterio investigado

\begin{tabular}{|c|c|c|c|c|c|c|c|c|c|c|c|c|c|c|c|c|}
\hline \multirow{2}{*}{\multicolumn{2}{|c|}{$\begin{array}{l}\text { Calificación de } \\
\text { cada Criterio } \\
\text { Criterios }\end{array}$}} & \multicolumn{2}{|c|}{$\begin{array}{l}\text { Defi- } \\
\text { ciente }\end{array}$} & \multicolumn{2}{|c|}{$\begin{array}{l}\text { Insufi- } \\
\text { ciente }\end{array}$} & \multicolumn{2}{|c|}{ Regular } & \multicolumn{2}{|c|}{ Bueno } & \multicolumn{2}{|c|}{ Excelente } & \multicolumn{2}{|c|}{$\begin{array}{c}\text { Total de } \\
\text { respuestas }\end{array}$} & \multirow{2}{*}{$\begin{array}{l}\text { n } \\
\text { que } \\
\text { con- } \\
\text { testa }\end{array}$} & \multirow{2}{*}{$\begin{array}{c}\% \\
\text { Bueno } \\
+ \text { Exce- } \\
\text { lente }\end{array}$} & \multirow{2}{*}{$\begin{array}{l}\text { Intervalo } \\
\text { de Con- } \\
\text { fianza } \\
(95 \%)\end{array}$} \\
\hline & & $\mathbf{n}$ & $\%$ & $\mathbf{n}$ & $\%$ & n & $\%$ & $\mathbf{n}$ & $\%$ & $\mathbf{n}$ & $\%$ & n & $\%$ & & & \\
\hline $\begin{array}{l}\text { Relevancia } \\
\text { de los } \\
\text { contenidos }\end{array}$ & $\begin{array}{l}2018 \\
2019 \\
\text { Total }\end{array}$ & $\begin{array}{r}5 \\
5 \\
10\end{array}$ & $\begin{array}{l}0,5 \\
0,3 \\
0,4\end{array}$ & $\begin{array}{r}9 \\
11 \\
20\end{array}$ & $\begin{array}{l}0,8 \\
0,7 \\
0,8\end{array}$ & $\begin{array}{l}49 \\
41 \\
90\end{array}$ & $\begin{array}{l}4,4 \\
2,8 \\
3,5\end{array}$ & $\begin{array}{l}238 \\
214 \\
452\end{array}$ & $\begin{array}{l}21,5 \\
14,5 \\
17,5\end{array}$ & $\begin{array}{r}805 \\
1.206 \\
2.011\end{array}$ & & & & & & \\
\hline $\begin{array}{l}\text { Exposición } \\
\text { respetuosa } \\
\text { y clara }\end{array}$ & $\begin{array}{l}2018 \\
2019 \\
\text { Total }\end{array}$ & $\begin{array}{r}6 \\
7 \\
13\end{array}$ & $\begin{array}{l}0,5 \\
0,5 \\
0,5\end{array}$ & $\begin{array}{r}17 \\
5 \\
22\end{array}$ & $\begin{array}{l}1,5 \\
0,3 \\
0,9\end{array}$ & $\begin{array}{l}54 \\
28 \\
82\end{array}$ & $\begin{array}{l}4,9 \\
1,9 \\
3,4\end{array}$ & $\begin{array}{l}200 \\
162 \\
362\end{array}$ & & & & & $\begin{array}{l}100 \\
100 \\
100\end{array}$ & & & \\
\hline $\begin{array}{l}\text { Organiza- } \\
\text { ción de la } \\
\text { información }\end{array}$ & $\begin{array}{l}2018 \\
2019 \\
\text { Total }\end{array}$ & $\begin{array}{r}11 \\
4 \\
15\end{array}$ & $\begin{array}{l}1,0 \\
0,3 \\
0,6\end{array}$ & $\begin{array}{r}6 \\
10 \\
16\end{array}$ & $\begin{array}{l}0,6 \\
0,7 \\
0,7\end{array}$ & $\begin{array}{l}55 \\
38 \\
93\end{array}$ & $\begin{array}{l}5,0 \\
2,6 \\
3,8\end{array}$ & $\begin{array}{l}253 \\
205 \\
458\end{array}$ & $\begin{array}{l}22,9 \\
14,1 \\
18,5\end{array}$ & $\begin{array}{r}781 \\
1.216 \\
1.997\end{array}$ & & & $\begin{array}{l}100 \\
100 \\
100\end{array}$ & $\begin{array}{l}221 \\
294 \\
515\end{array}$ & 93,5 & \\
\hline $\begin{array}{l}\text { Resumen } \\
\text { Calificación } \\
\text { de Conte- } \\
\text { nidos }\end{array}$ & $\begin{array}{l}2018 \\
2019 \\
\text { Total }\end{array}$ & $\begin{array}{l}3 \\
3 \\
6\end{array}$ & $\begin{array}{l}0,3 \\
0,2 \\
0,3\end{array}$ & $\begin{array}{r}10 \\
9 \\
19\end{array}$ & $\begin{array}{l}0,9 \\
0,6 \\
0,7\end{array}$ & $\begin{array}{l}44 \\
28 \\
72\end{array}$ & $\begin{array}{l}4,0 \\
1,9 \\
3,0\end{array}$ & $\begin{array}{l}299 \\
244 \\
543\end{array}$ & $\begin{array}{l}27,0 \\
16,7 \\
21,8\end{array}$ & $\begin{array}{r}750 \\
1.189 \\
1.939\end{array}$ & $\begin{array}{l}67,8 \\
80,6 \\
74,2\end{array}$ & $\begin{array}{l}1.106 \\
1.473 \\
2.579\end{array}$ & $\begin{array}{l}100 \\
100 \\
100\end{array}$ & $\begin{array}{l}221 \\
294 \\
515\end{array}$ & $\begin{array}{l}94,8 \\
97,3\end{array}$ & \\
\hline Promedios & & & & & & & $3 \%$ & & & $35 \%$ & & $2.579,8$ & 100 & $515^{*}$ & 95,4 & \\
\hline
\end{tabular}

* 515 de estudiantes que contestan de los 257 aprobados en 2018 y de los 344 aprobados en 2019 y su total de 601 estudiantes aprobados. Contesta el 85,7\%.

ción tradicional en la planificación familiar, en 1/4 de esta población estudiantil con IC de 20,2-27,2.

Son resultados preocupantes, para futuros profesionales con responsabilidades de resolver dudas, dar indicaciones, recomendar interconsultas y tratar con propiedad materias sensibles y privadas de conductas sexuales. En un país con escasa educación sexual integral escolar, (ESIE), las consecuencias se expresan en indicadores psicosociales y de salud sexual y reproductiva como ocurre en Chile. Surge la necesidad que las Facultades universitarias de carreras de la Salud, incluyan CFG en Educación Sexual. Especialmente en aquellas con mayor proporción estudiantes provenientes de la educación pública.

El cumplimiento de los objetivos de los 5 módulos del curso alcanzó 94,9\% en los criterios de Bueno y Excelente, con IC de 93,2-96,5\%. Los niveles de deficiencia e Insuficiencia variaron de $0,4 \%$ a $2,8 \%$ y las calificaciones de Regulares variaron de 2,9\% a 6\%. En el segundo semestre de 2019, la interrupción de actividades, no afectó la significación de los resultados. Este modelo On Line de Auto Aprendizaje, permitió al estamento estudiantil continuar sus actividades, pues todo el material docente estuvo desde el inicio disponible para su manejo, con evaluaciones automáticas y auto recuperaciones voluntarias de actividades. La comunicación On Line se mantuvo en forma permanente a solicitud de las y los estudiantes, para contestar sus preguntas, dudas, sugerencias y desacuerdos con los textos de capítulos y preguntas. Esto permitió avaluar el curso en forma constante. La experiencia docente más interesante se relaciona con los temas de Desarrollo Psicosexual, Género, Aborto, Transexualidad y Respuesta Sexual Humana que acumularon la mayor cantidad de dudas, desacuerdos y sugerencias. Pero la materia de Diversidad Sexual se percibe que aun no es tratada suficientemente para cubrir un amplio espectro de este tema.

La medición de la Calidad del Desarrollo del Curso se basó en 4 criterios pedagógicos definidos por la Facultad. Las calificaciones de cada uno, tuvo un promedio de 95,35\% de Bueno y Excelente con IC de $91,4 \%$ a $98,1 \%$. No hubo temas o materias objetados.

La aceptación docente fue muy alta, a pesar 
que sólo 10\% de los estudiantes participantes los Foros Virtuales, preguntan. La materia curricular tratada con esta metodología sido un aporte positivo a la docencia, donde hay alto consumo de tiempo en disciplinas con mucha exigencia y dedicación presencial prioritaria e indispensable para la adquisición de habilidades.

Este modelo en aspectos básicos de la sexualidad, permitiría profundizarlas en cursos posteriores, de acuerdo a la experiencia y subespecialidades de cada Carrera. En Medicina, se podrá profundizar en materias de Sexología Humana, en la persona sana o con morbilidades y ser la base para el desarrollo de la subespecialidad derivada en Sexología Humana. Además permite a la generación de nativos digitales, programar sus tiempos y ocupar aquellos períodos de inactividad docente.

En el primer semestre 2020, este CFG fue replicado a 989 estudiantes a solicitud de la Facultad de Medicina y otras Facultades, dado que muchos CFG presenciales, no se desarrollaron debido a la pandemia COVID-19. Aprobaron 897 estudiantes (90,7\%). El modelo docente respondió técnica y didácticamente. Cabe mencionar que el mismo modelo, replicado en una plataforma de Cursos Abiertos $^{34}$ ha sido instalado por las Direcciones Estudiantiles de las Universidades de Antofagasta y La Serena, con éxito en su funcionamiento desde 2018.

Es un gran aporte de capacitación a Profesores de enseñanza Básica, Media, Técnica, Parvularias, docentes de Primer nivel y apoyo a madres, padres y apoderadas $/ \mathrm{os}^{39}$, contribuyendo al desarrollo de la ESIE recomendada por UNESCO ${ }^{29}$, como ocurre en todos los países desarrollados y en la mayoría de los países del mundo en desarrollo.

Agradecimientos: Se expresa el reconocimiento al equipo que ha contribuido en el desarrollo Virtual de CESOLAA con su material, donado a la Universidad de Chile a través de la DIBAM y a las/os docentes que han colaborado en las clases virtuales actualizando los temas: Alejandro Afani, Fanny Berlagosky, Felipe Calderón, Daniela Castro, Magdalena Castro, Pamela Eguigüren, Germán Jara, Carolina Leyton y Victoria Paredes, Paulina Troncoso y al apoyo del equipo de la UTIE de la Escuela de Salud Pública: Jorge Caro Miranda, David Euluffi, Alex Benavides Soto y Paola Videla Lagos.

\section{Referencias}

1. UNESCO, 2018, International technical guidance on sexuality education:Introduction page 12 , Section 4 : The evidence base for comprehensive sexuality education, pages 28-31. http://www.unaids.org/sites/default/files/ media_asset/ITGS E_en.pdf.

2. United Nations. Report of the International Conference on Population and Development, Document A/ Conf. 171/13, New York, , 1994, paragraph 7.2., Kirby D, Rolleri L and Wilson MM. 2007. Tool to Assess the Characteristics of Effective Sex and STD/HIV Education Programmes. Washington, DC, Healthy Teen Network. Kirby, D., Rolleri, L. and Wilson, M. M. 2007. https://www.google.com/search?q=Kirby,+D.,+Rolleri, + L.+and+Wilson, + M.+M.+2007.+Tool+to+Assess + the + Characteristics + of + Effective + Sex + and + STD/ $\mathrm{HIV}+$ Education + Programmes. + Washington, $+\mathrm{D}-$ $\mathrm{C},+$ Healthy + Teen + Network\&ie $=$ utf- $8 \&$ oe $=$ utf-8\&clien $\mathrm{t}=$ firefox-b-ab\&gfe_rd $=$ cr\&dcr=0\&ei=FImYWpnaIYGfXsnvlNAN

3. World Health Organization . Journal Paper. Sexual and Reproductive Health: a matter of life and death. Glase A, Güemezoglu AM, Schmid GP, García Motreno C, Van Look FA. The Lancet Sexual and Reproductive Health Series, October 2006.

4. Rohrbach LA, Berglas NF, Jerman P, Angulo-Olaiz F, Chou CP, Constantine N. A Rights-Based Sexuality Education Curriculum for Adolescents: 1-Year Outcomes From a Cluster-Randomized Trial. J Adolescent Health 2015; 57(4): 399-406.

5. UNESCO 2016. Review of the Evidence on Sexuality Education. Report to inform the update of the UNESCO International Technical Guidance on Sexuality education; prepared by Paul Monygomery and Wendy Knerr, University of Oxford Centre for Evidence-Based Intervention.

6. UNESCO 2009. International Technical Guidance on Sexuality Education: An Evidence-informed approach for schools, teachers, and health educators. Paris. UNESCO. https://www.unaids.org/sites/default/files/media_asset/ ITGSE_en.pdf.

7. Ley 20.418. https://www.google.com/search?client=firefox-b-e\&q=Ley+20.418.

8. Reglamento Ley 20.418. https://www.google.com/search?client=firefox-b-e\&q=Reglamento+Ley+20.418.

9. Castro G, Carrasco M, Solar F, Catrien M, Garcés C, Marticorena C. Impacto de las políticas de educación sexual en la salud sexual y reproductiva adolescente en el sur de Chile, período 2010-2017. Rev Chil Obstet Ginecol 2019; 84 (1): 28-40. https://scielo.conicyt.cl/ 
scielo.php?sc3ipt=sci_arttext\&pid=S0717-75262019000 100028\&lng=es\&nrm=iso.

10. Boletín 12593-04 Cámara de Diputados de Chile 25 abril 2019. Modifica la Ley 20.418 con el objeto de perfeccionar las disposiciones de la ley, en particular en lo relativo a la educación en materia de sexualidad, afectividad y género. https://www.google.com/search?client=firefox-b-e\&q=Bolet $\%$ C3\%ADn+12593-04+C\%C3\%A$1 \mathrm{mara}+\mathrm{de}+$ Diputados $+\mathrm{de}+\mathrm{Chile}+25+$ abril $+2019 .+\mathrm{Mo}-$ difica+la+Ley+20.418+con+el+objeto+de+perfeccionar+las+disposiciones + de + la + ley $\% 2 C+e n+$ particular+en+lo+relativo $+\mathrm{a}+\mathrm{la}+\mathrm{educaci} \% \mathrm{C} 3 \% \mathrm{~B} 3 \mathrm{n}+\mathrm{en}+\mathrm{ma}-$ teria + de + sexualidad $\% 2 \mathrm{C}+$ afectividad $+\mathrm{y}+\mathrm{g} \% \mathrm{C} 3 \% \mathrm{~A}-$ 9nero.t

11. Sesión $\mathrm{N}^{0}$ 161, Junio de 2020, Comisión de Educación de la Cámara de Diputados, Primer Trámite constitucional Reforma a Ley 20.418. Sesión N0 89 Especial,15 de octubre 2020. Cámara de Diputado de Chile No Aprueba reforma a ley 20.418. https://www.google. $\mathrm{com} /$ search?client=firefox-b-e\&q=camara + de+diputados+ley+de+educaci $\%$ C3\%B3n+sexual

12. Síntesis Histórica de la Universidad de Chile http://web. uchile.cl/historia/historia.html.

13. Ortiz-Moreira LE, Fasce-Henry EA, Matus-Betancourt OB, Campos CI. Educación médica y diversidad cultural en Chile. Un diálogo pendiente. Rev Med Chile 2018;146:922-928. https://scielo.conicyt.cl/pdf/rmc/ v146n7/0034-9887-rmc-146-07-0922.pdf.

14. Montero VA. Educación sexual: un pilar fundamental en la sexualidad de la adolescencia. Rev Med Chile 2011; 139: 1249-1252. http://www.scielo.cl/scielo.php?pi$\mathrm{d}=$ S0034-98872011001000001\&script=sci_arttext.

15. Johnson K, Rullo J, Faubion S. Student-Initiated Sexual Health Selective as a Curricular Tool. Sex Med 2015; 3 (2): 118-27.

16. Komlenac N, Siller H, Hochleitner M. Medical Students Indicate the Need for Increased Sexuality Education at an Austrian Medical University. Sex Med 2019; 7 (3): 318-25.

17. Criniti S, Andelloux M, Woodland MB. The state of sexual health education in U.S. medicine. Am J Sex Educ 2014; 9: 65-80.

18. Wight $\mathrm{D}$. The effectiveness of school-based sex education: What do rigorous evaluations in Britain tell us? Education and Health 2011; 29 (4): 72-8. https://www. google.com/search?q=Wight,+D.+2011.+The+effectiveness+of+schoolbased+sex+education:+What+do+rigorous+evaluations + in + Britain + tell + us $\% 3 F+$ Education + and + Health, $+29(4),+72-78$. $) . \& i e=u t f-8 \& o e=u-$ tf-8\&client=firefox-b-ab\&gfe_rd $=$ cr\&dcr $=0 \&$ ei $=$ vouYWqr_DY6fXrv8lcgC.
19. Shindel AW, Parish SJ. CME information: Sexuality education in North American medical schools: Current status and future directions. J Sex Med 2013; 10 (1): 3-17 quiz 18. doi: 10.1111/j.17436109.2012.02987.x.

20. Zamboni B, Bezek K. Medical students' perceptions and preferences for sexual health education. J.Sex Educ-Sex Soc Learn 2017; 17: 371-85.

21. Warner C, Carlson S, Crichlow R, Ross MW. Sexual Health Knowledge of U.S. Medical Students: A national survey. J Sex Med 2018; 15 (8): 1093-102. doi: 10.1016/j. jsxm.2018.05.019. Epub 2018 Jul 13.

22. Rufino AC, Madeiro A, Girão MJBC. Sexuality education in Brazilian medical schools. J Sex Med 2014; 11: 1110-7.

23. Turner D, Jopt K, Nieder TO. German medical students' interest in and knowledge about human sexuality in 1972 and 2012. J Sex Med 2014; 11: 1914-26.

24. Prof. Waldemar Coutts. Memoria Chile. Biblioteca Nacional de Chile. http://www.memoriachilena.gob.cl/602/ w3-propertyvalue-128849.html.

25.- Prof. Dr. Osvaldo Quijada Cerda http://alejandro-rios-rojas.blogspot.com/2014/03/profesor-drosvaldo-quijada-cerda.html.

26. Jara G, Molina R. Educación Sexual . Guía para Educadores. Ed. Arancibia 1993.

27. Molina R. Educación Sexual escolar .EN: Enfoque actual de la Adolescente por el ginecólogo. Ed Prof. José María Méndez Ribas, ed. ASCUNE, Buenos Aires, 2004.

28. Molina T, González E, Jara G, Molina R. Elaboración y Validación de un programa piloto continuo interactivo de educación sexual a través de Internet. R.Ch.Obstet Gineclo Inf Adolec 2005; 12: 71-7.

29. UNESCO. 2009. International Technical Guidance on Sexuality Education: An Evidence-informed approach for schools, teachers and health educators. Paris, UNESCO https://www.google.com/search?client=firefox-b-e\&q=UNESCO.+2009.+International+Technical+Guidance+on+Sexuality+Education $\% 3 \mathrm{~A}+\mathrm{An}+$ Evidence-informed+approach+for+schools $\% 2 \mathrm{C}+$ teachers+and+health+educators.+Paris\%2C+UNESCO+

30. Molina T, González E, Jara G, Ortiz C, Castillo T, Molina R, et al. Elaboración y Validación de un Programa piloto de apoyo continuo interactivo de Educación Sexual a través de Internet, destinados a docentes capacitados en Educación Sexual en CEMERA: Parte II Ejecución Programa Interactivo Rev Soc Ch Obstet \& Ginecol Inf. y Adolec 2005; 12 (1): 25-32. https://www.google.com/search?client=firefox-b-d\&q=Molina+T\%2C+Gonz\%C3\%Allez+E.\%2C+Jara+G.\%$2 \mathrm{C}+$ Ortiz+C. $\% 2 \mathrm{C}+\mathrm{Del}+\mathrm{Cas}$ tillo+T\%2C+Lorca+A. $\%-$ 
$2 \mathrm{C}+$ Molina + R. $\% 2 \mathrm{C}+$ Fresia + C. $+\%$ E2\%80\%9CElaboraci\% $\mathrm{C} 3 \% \mathrm{~B} 3 \mathrm{n}+\mathrm{y}+$ validaci $\% \mathrm{C} 3 \% \mathrm{~B} 3 \mathrm{n}+\mathrm{de}+\mathrm{un}+$ programa+piloto+de+apoyo+continuo+interactivo+de+educaci $\% \mathrm{C} 3 \% \mathrm{~B} 3 \mathrm{n}+$ sexual $+\mathrm{a}+$ trav $\% \mathrm{C} 3 \% \mathrm{~A} 9 \mathrm{~s}+\mathrm{de}+$ Interne$\mathrm{t} \% 2 \mathrm{C}+$ destinados+a+docentes+capacitados+en+educaci\%C3\%B3n+.

31. Apter D, Molina CR. Sexuality Education: Finnish and Chilean Experiences En: Sultan C (ed): Pediatric and Adolescent Gynecology. Evidence- Based Clinical Practice. 2nd, revised and extended edition. Endocr Dev Basel, Karger 2012; 22: 332-56

32. Molina R. Educación Sexual escolar. En: Enfoque actual de la Adolescente por el ginecólogo. Ed Prof. José María Méndez Ribas, ed. ASCUNE, Buenos Aires, 2015.

33. Libro de Educación Sexual On Line de Auto Aprendizaje. CESOLAA. Pág. 204 http://www.educacionsexual. uchile.cl/libro/\#page/407.

34. Curso de Educacional sexual On Line de Auto Aprendizaje. www.educacionsexual.uchile.cl.

35. Elaboración de Tests. Desarrollo e interpretación de los test de aprovechamiento. Capitulo 9. Análisis de estímulos. Dificultad de los reactivos (pag. 102-15). Dorothy Adkins Wood. Quinta Reimpresión en Español, febrero 1975. Editorial Trillas, S.A. México. Av. 5 de Mayo 43105 México 1, D.F.

36. Toledo V, Luengo X, Molina R, Murray N, Molina T, Villegas R. Impacto del programa de educación sexual: Adolescencia ,tiempo de decisiones. En: Capítulo 56, Salud Sexual y Reproductiva en la Adolescencia, R. Molina, J. Sandoval y E. González. 2003 editorial Mediterraneo Ltda. ISBN 956-220-219-4. https://www.google.
com/search?q=Virginia + Toledo $\% 2 \mathrm{C}+\mathrm{Ximena}+$ Luengo $\% 2 \mathrm{C}+$ Ramiro+Molina $\% 2 \mathrm{C}+\mathrm{Nancy}+$ Murray $\% 2 \mathrm{C}-$ Temistocles + Molina $+\mathrm{y}+$ Rodrigo + Villegas. + Impacto +del+programa + de + educaci $\% \mathrm{C} 3 \% \mathrm{~B} 3 \mathrm{n}+$ sexual $\% 3 \mathrm{~A}+\mathrm{A}-$ dolescencia $+\% 2 \mathrm{Ctiempo}+$ de + decisiones. + En $+\% 3 \mathrm{~A}+-$ Capitulo $+56 \% 2 \mathrm{C}+\mathrm{Salud}+\mathrm{Sexual}+\mathrm{Y}+$ Reproductiva+en+la+Adolescencia $\% 2 \mathrm{C}+\mathrm{R} .+$ Molina $\% 2 \mathrm{C}+\mathrm{J} .+-$ Sandoval+y+E.+Gonz\%C3\%A1lez.+2003+editorial+Mediterraneo+Ltda. + ISBN+956-220-219-4\%29\&ie $=\mathrm{u}-$ tf-8\&oe=utf-8\&client=firefox-b-ab.

37. Jara G. Educación Sexual: Experiencias con profesores. En: Capítulo 55, Salud Sexual y Reproductiva en la Adolescencia, R. Molina, J. Sandoval y E. González. 2003 editorial Mediterráneo Ltda. ISBN 956-220-219-4. https://www.google.com/ search?client=firefox-b-e\&q=Germán+Jara. + Educación+Sexual $\% 3 \mathrm{~A}+$ Experiencias + con + profesores.+En $\% 3 \mathrm{~A}+$ Capítulo $+55 \% 2 \mathrm{C}+\mathrm{Salud}+\mathrm{Sexual}+\mathrm{y}+\mathrm{Re}-$ productiva + en + la + Adolescencia $\% 2 \mathrm{C}+\mathrm{R} .+$ Molina $\%$ 2C+J.+Sandoval+y+E.+González.+2003+editorial+Mediterráneo+Ltda.+ISBN+956-220-219-4.

38. Principles of Medical Statistics. Confidence Limits.(page 141-142) Bradford Hill. Eight edition Reprinted 1967. OXFORD UNIVERSITY PRESS. Copyright The Lancet Ltd. 1966. Printed in Great Britain by R.\& Clark. LTD. Edinburgh.

39 Centro de Perfeccionamiento, Experimentación e Investigaciones Pedagógicas. Ministerio de Educación. Certifica Universidad de Chile. Curso de Educación Sexual On Line de Auto Aprendizaje. Alcance Nacional. Resolución Exenta No 4133 del 21.08.2019. 